UJIIÉ REPLIES-The Cretaceous kerogen of the Douala Basin is the best material available to provide a corroborative evidence of my hypothesis on the reversal in the $\mathrm{C}-\mathrm{H}-\mathrm{O}$ maturation pathway of kerogen, because the Cretaceous is composed of the thick argillaceous sedimentary series in which the original organic matter is identical throughout the whole cores taken between 700 and $4,000 \mathrm{~m}$ in depth ${ }^{1,2}$.

Jones concluded that the anomalous point $(g)$ had a different type kerogen. A series of geochemical studies on the organic matter of the Douala Basin indicates the same kind of organic matter provided in the sample $(g)$ as in the other ones $^{1-3}$. Tissot et al. ${ }^{4}$ pointed out that the whole evolution path of these kerogens is the typical example of their 'type III'.

In order to examine the original kerogen type the maceral components of kerogen should be discussed, instead of the minor difference of hydrogen content and weight loss by pyrolysis as was stated by Jones. The maceral component of each kerogen of the Douala Basin almost consist of more than $80 \%$ of amorphous organic cement optically close to vitrinite $^{1}$, indicating the homogeneous components throughout the whole cores. Therefore, the $\mathrm{C}-\mathrm{H}-\mathrm{O}$ component path from $(g)$ to $(h)$ should be explained by a large amount of hydrocarbon release, not by the difference of original kerogen type in $(g)$.

Kerogen is extracted organic matter. Its $\mathrm{C}-\mathrm{H}-\mathrm{O}$ content should be related to what kinds of the generated products are released from it, if the original kerogen is identical throughout the strata. The same reversal in the $\mathrm{C}-\mathrm{H}-\mathrm{O}$ maturation pathway as shown from $(g)$ to $(h)$ in the Douala Basin is observed in the Miocene argillaceous sediments of the MITI Borehole in Northern Hokkaido. Examination of hydrocarbon-generating capacity by pyrolysis supports that the relative decrease of carbon content is due to the hydrocarbon release in the MITI Borehole samples (data to be published elsewhere). Therefore, kerogen analysis could give us much information on petroleum generation. To determine whether my hypothesis is applicable to the Douala Basin, the most important work to be done is to decide whether the weight loss by pyrolysis at $(g)$ is due to the generation mainly of hydrocarbon or mainly of $\mathrm{CO}_{2}$ and/or $\mathrm{H}_{2} \mathrm{O}$.

\section{YOSHIHIRO UJIIÉ}

Department of Geology and Mineralogy,

Faculty of Science,

Hokkaido University,

Sapporo, 060, Japan

1. Durand, B. \& Espitalié, J. Geochim. cosmachim. Acta 40 , 801-808 (1976)

2. Albrecht, P., Vandenbroucke, M. \& Mandengué, M Geochim. cosmochim. Acta 40, 791-799 (1976).

3. Vandenbroucke, M., Albrecht, P.\& Durand, B. Geochim cosmochim. Acta 40, 1241-1249 (1976).

4. Tissot, B., Durand, B., Espitalić, J. \& Combaz, A. Bull. Am. Ass. petrol. Geol. 58, 499-506 (1974).

\title{
Stability problem of Kirkwood Gaps
}

KIANG noticed ${ }^{1}$ the importance of the Kirkwood Gaps (particularly the Hecuba Gap) in contrast to Hilda Group in the asteroidal distribution problem, and presented a solution to this problem using a stability criterion inherited in Hill's equation which, he assumed, clarifies the difference between gaps and groups (less populated against populated regions). His discussion, however, discloses the curious fact that he has omitted the first two terms in RHS of his (linearised) variational equation (6), without any convincing ground. This omission causes a critical deviation from the original problem.

Kiang's discussion starts essentially from the canonical equations of motion with one degree of freedom,

$$
\frac{\mathrm{d} x}{\mathrm{~d} t}=F_{\mathrm{y}} \quad \text { and } \quad \frac{\mathrm{d} y}{\mathrm{~d} t}=-F_{x}
$$

where the subscripts refer to the partial derivatives with respect to the variables $x$ and $y$, respectively. Let $x(t)$ and $y(t)$ be periodic. Then he deduces the variational equations,

and

$$
\left.\begin{array}{l}
\frac{\mathrm{d} \xi}{\mathrm{d} t}=F_{y x} \xi+F_{y y} \eta \\
\frac{\mathrm{d} \eta}{\mathrm{d} t}=-F_{x x} \xi-F_{x y} \eta
\end{array}\right\}
$$

where $\xi$ and $\eta$ are the virtual variations to $x$ and $y$ coordinates, respectively.

$\mathrm{He}$ insists that from these variational equations he obtained a second order differential equation of Hill's type. Curiously enough, however, the system of equations (2) can be solved by quadrature as will be seen by equations (9), as it has a (variational) energy integral (see also ref. 2)

$$
\mu \equiv F_{x} \xi+F_{y} n=c_{1}=\text { constant }
$$

This $\mu$ is a variation across the orbit. We also have a variation along the orbit by a form,

$$
\nu \equiv F_{y} \xi-F_{x} \eta
$$

which is perpendicular to $\mu$ direction. The differential equation for $\nu$ can be obtained by a straightforward manipulation as follows:

$$
\frac{\mathrm{d} \nu}{\mathrm{d} t}=(A \mu+B \nu) / D
$$

where

$$
\left.\begin{array}{l}
A=\left(F_{x x}-F_{y y}\right)\left(F_{x}^{2}-F_{y}^{2}\right)+4 F_{x y} F_{x} F_{y} \\
B=-2 F_{x y}\left(F_{x}^{2}-F_{y}^{2}\right)+2\left(F_{x x}-F_{y y}\right) F_{x} F_{y}
\end{array}\right\}
$$

and

$$
D=F_{x}^{2}+F_{y}^{2} \neq 0
$$

On the other hand, we see

$$
\frac{\mathrm{d} D}{\mathrm{~d} t}=B
$$

Therefore, we can obtain the solution for $\nu$ by quadrature, considering that $\mu$ is given by equation (3),

$$
\nu=D\left[c_{2}+c_{1} \int_{0}^{t} D^{-2} A \mathrm{~d} t\right]
$$

$c_{2}$ being another constant. The solutions for $\xi$ and $\eta$ are readily given by

$$
\left.\begin{array}{l}
\xi=c_{1} F_{x} D^{-1}+F_{y}\left[c_{2}+c_{1} \int_{0}^{t} D^{-2} A \mathrm{~d} t\right] \\
\text { and } \\
\eta=c_{1} F_{y} D^{-1}-F_{x}\left[c_{2}+c_{1} \int_{0}^{t} D^{-2} A \mathrm{~d} t\right]
\end{array}\right\}
$$

Note that the solutions by quadrature do not include a new period as in the case of Hill's equation, although the solutions include the mixed secular term, $t \times$ (periodic term) in general. This situation comes from the change of period according as different energy constants (the energy difference being $c_{1}$ ).

Kiang's argument is totally wrong in the point: he only took into account some of the necessary terms without any convincing ground, so that the equation becomes the Hill's type. In reality, the variational equations (2) can be solved by quadrature, because the system has an energy integral. There is no need to introduce a transcendental method, or a new period. In other words, the stability criterion, with which he wanted to clarify the difference between Hecuba and Hilda cases, is not inherent in the variational equations (2) $a b$ initio. Thus the problem is still open.

$$
\text { S. AOKI }
$$

Tokyo Astronomical Observatory, Mitaka, Tokyo,

\section{Japan}

1. Kiang, T. Nature 273, 734-736 (1978).

. Dilliberto, S. P. in Contribution to the Theory of Non-linear Oscillations Vol. 1 (ed. Lefschetz, S.) 1-38 (Princeton University Press, 1930).

KIANG REPLIES-Aoki's attitude is the conventional one and is responsible for the lack of success in solving the Kirkwood Gaps problem so far. He apparently believes that because he could write down the solution of the basic equations by the classical method, there is no need to introduce other methods. But the classical method is, in principle, incapable of solving the problem at hand, because it always leads to results (see, for example, Aoki's equations 9) that apply to all resonances equally-it can never explain the HecubaHilda difference. Hence I regard it highly desirable to introduce some new method, equivalent to more or less radical departures from the classical method. Some modification to explain the Hecuba-Hilda difference within the frame of the simplest gravitational model is necessary but as to the form of modification I adopted in my paper, I made it clear that there may well be grounds for improvement. I do not expect any improvement that I could give here to substantially alter the result obtained.

\section{T. KIANG}

Dunsink Observatory, Castleknock, Co. Dublin, Ireland 